\title{
The Editor Emeritus: Celebrating Dr. Laura Roberts
}

\section{Adam M. Brenner ${ }^{1}$ (D) $\cdot$ Richard Balon $^{2} \cdot$ Eugene V. Beresin $^{3} \cdot$ John Coverdale ${ }^{4} \cdot$ Anthony P. S. Guerrero $^{5}$. Alan K. Louie $^{6} \cdot$ Rashi Aggarwal $^{7} \cdot$ Mary K. Morreale $^{2}$}

Received: 19 September 2019 / Revised: 19 September 2019 / Accepted: 24 September 2019/Published online: 11 November 2019

(C) Academic Psychiatry 2019

The December 2019 issue of Academic Psychiatry is the last featuring papers largely received and developed during the remarkable tenure of Dr. Laura Roberts as Editor in Chief. This is therefore an ideal, if bittersweet, opportunity to celebrate her vision, leadership, and many accomplishments.

Dr. Roberts stepped into the role in 2002. With 17 years at the helm, she has been the longest tenured Editor in Chief of Academic Psychiatry. To put this in perspective, in 2002, the world was still 2 years away from the launch of Facebook, and Apple would not sell the first iPhone for another 5 years. We were also 1 year away from the establishment of resident dutyhour limits, and it would be 11 more years before the Accreditation Council for Graduate Medical Education introduced the milestones project. Many of our readers may have spent their entire careers, and sometimes even their training, in the era of Dr. Roberts' leadership. To appreciate this from another perspective, the journal has been in existence since 1977 (beginning in its earlier incarnation as the Journal of Psychiatry Education). Dr. Roberts served as Editor in Chief for $40 \%$ of the journal's lifespan to date. This longevity itself is an accomplishment and an inspiration. At a time when we are increasingly aware of the danger of burnout for academic psychiatry faculty, Dr. Roberts provides a model for sustained passion and generativity.

Adam M. Brenner

adam.brenner@utsouthwestern.edu

1 University of Texas Southwestern Medical Center, Dallas, TX, USA

2 Wayne State University, Detroit, MI, USA

3 Harvard Medical School, Boston, MA, USA

4 Baylor College of Medicine, Houston, TX, USA

5 University of Hawai'i John A. Burns School of Medicine, Honolulu, HI, USA

6 Stanford University, Stanford, CA, USA

7 Rutgers New Jersey Medical School, Newark, NJ, USA
Among Dr. Roberts' earliest achievements as editor was the addition of two new sponsoring organizations, the Association of Directors of Medical Student Education in Psychiatry (ADMSEP) in 2003 and the American Association of Chairs of Departments of Psychiatry (AACDP) in 2005. They joined the American Association of Directors of Psychiatric Residency Training (AADPRT) and the Association for Academic Psychiatry (AAP), the organizations that had supported the journal since its inception. In hindsight, it is easy to think of this expansion as inevitable, especially given how well the culture and missions of ADMSEP and AACDP fit with the journal's scope and mission and how smoothly the four organizations' consortium works together. But in reality, this accomplishment is very rare.

When the incoming Editor in Chief attended a recent conference of science journal editors, he learned that journals cosponsored by two organizations were unusual and that cosponsorship by four organizations was obviously impossible to coordinate. Too often, issues of control and differences in the mission and vision of stakeholder organizations come into conflict when collaboration even in common scientific enterprises is on the table. The success of this arrangement is in large part due to Dr. Roberts' gift for bringing people and institutions together. As many readers can attest, getting one national organization to agree on an agenda is itself an accomplishment!

How did Dr. Roberts achieve this? One reason is that she approaches every individual member and the leadership of the four sponsoring organizations with great respect and positivity. She expects the same collegiality in how individuals and organizations work with each other.

Another reason is that members of each organization could expect to find Dr. Roberts at their annual meeting, where they met an editor who was clearly open to their individual feedback, committed to their development, and deeply appreciative of the support of their association. Each organization felt that she was truly a part of their team and clearly appreciated that she was attentive to the audience they addressed. She 
listened. She learned from them, and she valued addressing their interests in the journal. In short, she developed a trusting relationship such that one common journal could address the many, and, at times, disparate, issues in psychiatric education.

She also introduced the tradition of herself or other editors presenting workshops on writing and reviewing at each organization's meeting to help participants publish and understand the process of publishing. She especially cultivated the enthusiasm and skills of early career and first-time authors. In her workshops, she also addressed the particular challenges facing women and provided sage advice on how to navigate them.

She ensured and expanded the tradition that was initially established by former editor Jon Borus and his editorial team of the peer reviewers themselves being educational guides for authors. Every author received feedback, sometimes quite detailed, about their submission. For leaders of the stakeholder organizations, this practice meant that not only was evidencebased material produced but the journal also could serve as a means for professional development.

Readers will not be surprised to learn that Dr. Roberts grew the journal during her tenure, with a track record of remarkable goals and accomplishments that have been outlined in previous editorials [1-7]. The extent of that growth may still be a revelation, however. When she assumed her role, the journal received approximately 100 submissions each year. In 2019, to date, we are on track for close to 400 new submissions. In 2002, the journal published a total of 268 pages; in 2018 , that number was 877 , which represents growth of over $20 \%$ per year - an impressive record in any enterprise! In 2005 , this growth reached an important tipping point, and the journal was able to move from quarterly to bimonthly publication.

The impact factor of a journal measures the frequency with which its articles are cited. It is calculated by the number of citations to articles in a given year divided by the number of substantive articles published in the journal over the previous 2 years. The impact factor is not a perfect or absolute measure - it captures only one aspect of a journal's cumulative influence. The impact factor for Academic Psychiatry was first calculated at 0.4 in 2002, Dr. Roberts' first year as Editor in Chief. Through her tenure, the impact factor has continued to rise to our most recent score of 1.88 for 2018 .

Another way to think about influence is how often papers are read. Many readers of Academic Psychiatry have praised the journal for the readability and practical application of its articles. The increasing use of the journal's website by readers allows us a quantitative window into this use. We have no idea how many recipients of the print journal actually open it and how many papers they read, but we are able to track this information for our online publication, and the results under Dr. Roberts' leadership are impressive. In 2014, readers downloaded the full text of articles in the journal 37,406 times. In 2018 , that number had grown to 169,178 .
In addition to the growth in raw numbers, Dr. Roberts also led the journal to growth in the variety of manuscript formats that we reviewed. Under her leadership, the journal created the Literary Resources column, Educational Case Reports, the Day-in-the-Life series, and the "Down to Earth" Academic Skills column. At the same time that the journal was increasing in variety and in volume, Dr. Roberts also managed to increase its efficiency. The last 3 years have seen the journal cut its time from submission to first decision by just over half. In 2015, there were an average of 52 days from receipt of the manuscript by the journal to communication of the first decision to the authors. In 2018, that turnaround time was down to 23 days.

In 2014, Dr. Roberts led one of the most significant changes in the journal since its inception. She successfully negotiated a new publishing home for Academic Psychiatry, moving from American Psychiatric Publishing, our publisher since the journal's birth in 1989, to Springer International Publishing AG. In this new relationship, the journal has continued to increase the number of institutions around the world that hold subscriptions to the journal and to extend its reach significantly. We are able to publish papers online within a month from acceptance, well ahead of when the print copy would appear. For academic authors whose professional standing and career promotion depend on publication, this swift timing can be of great value.

The transfer of Academic Psychiatry to Springer allowed the journal to achieve another of Dr. Roberts' goals: greater global outreach. Dr. Roberts has been interested in reaching educators in other countries to increase the exchange of information and experience. The journal started to publish more articles from other countries relevant to academic psychiatry and, to date, has received submissions from 74 different countries. The number of international submissions increased so much that the journal has now published contributions from all continents but Antarctica! The journal receives reviews regularly from international peer reviewers, and international members of the Editorial Board were added to increase its global visibility.

Another of Dr. Roberts' signature accomplishments is harder to measure: her creation of a generative and supportive culture at the journal. She actively encourages reviewers and editors to remember that the journal is not only a vehicle for scholarship but also a critical potential venue for professional development of the authors. In this light, she encourages the identification of positive aspects of papers received and their importance and potential contribution to the available literature along with a thoughtful and deep appraisal of how a manuscript could be improved. This approach is not limited to Dr. Roberts' writing or her work behind the scenes as an editor. She has also conveyed these values through her regular presence and approachability at the annual meetings of the four sponsoring organizations and her very popular 
workshops on writing for publication and career development. In addition, she has been a strong advocate for residents, fellows, and medical students to serve as authors and encouraged editorial board members to serve as mentors to new authors. Dr. Roberts is a champion of professional development from medical students to senior attending psychiatrists.

Dr. Roberts fostered a similar formative attitude among the editorial group. The assistant, associate, and deputy editors all benefited from her direct mentorship. Some of us progressed from first time authors, to first time reviewers, to editorial board members, and to our current roles, all under Dr. Roberts' leadership and guidance. In fact, every member of the current editorial group save one (Dr. Beresin) was recruited by Dr. Roberts. When each of us joined the editorial group, we were welcomed into a culture of creative thinking, lively discussion, productive disagreement, and warm camaraderie. This culture is an extension of who Dr. Roberts is as a scholar and a person. It is a part of the explanation for the journal's great success in retaining its volunteer editorial group.

Dr. Roberts clearly led the journal from a solid but small publication to a real global player in psychiatric education, a great achievement. Our editors' group, the membership of the sponsoring organizations (AACDP, AADPRT, AAP, and ADMSEP), and all those who value excellence in psychiatry education owe Dr. Roberts our admiration and gratitude. This transition provides us an opportunity to reflect on all we have learned from Dr. Roberts and to recommit ourselves to the values that she models and champions.

\section{Conflict with Ethical Standards}

Disclosure On behalf of all authors, the corresponding author states that there is no conflict of interest.

\section{References}

1. Roberts LW. Your journal, our journal. Acad Psychiatry. 2002;26:13.

2. Roberts LW, Coverdale J, Louie A, Beresin EV. Academic psychiatry: coming of age. Acad Psychiatry. 2005;29:237-9.

3. Coverdale J, Roberts LW, Louie A, Beresin EV. Enhancing the international status of academic psychiatry. Acad Psychiatry. 2007;31: 177-9.

4. Coverdale J, Roberts LW, Balon R, et al. Improving the "impact" of Academic Psychiatry. Acad Psychiatry. 2008;32:169-72.

5. Balon R, Roberts LW, Coverdale J, et al. Globalization of medical and psychiatric education and the focus of Academic Psychiatry on the success of "international" authors. Acad Psychiatry. 2008;32: 151-3.

6. Roberts LW, Coverdale J, Balon R, et al. In celebration of the history of Academic Psychiatry. Acad Psychiatry. 2013;37:369-74.

7. Balon R, Beresin EV, Coverdale J, et al. A new day for Academic Psychiatry. Acad Psychiatry. 2014;38:1-4.

Publisher's Note Springer Nature remains neutral with regard to jurisdictional claims in published maps and institutional affiliations. 